\title{
On a Steady-State Quantum Hydrodynamic Model for Semiconductors
}

\author{
Bo Zhang*and Joseph W. Jerome ${ }^{\dagger}$
}

August, 1994

\begin{abstract}
A third order quantum perturbation of the stress tensor, and a relaxation approximation to represent averaged collisions, are employed as perturbations of the isentropic model for a collisionless plasma. The model is self-consistent in the sense that the electric field, which forms a forcing term in the momentum equation, is determined by the coupled Poisson equation. As formulated, the model is a reduced version of the quantum hydrodynamic model for semiconductors. Existence is demonstrated for the model, which is shown to be equivalent to a non-standard integro-differential equation. An unusual boundary condition, with the important physical interpretation of specifying the quantum potential at the (current) inflow boundary, is identified as essential for the theory.
\end{abstract}

Key words: Quantum hydrodynamic model, integro-differential equation, quantum perturbation AMS(MOS) Subject Classification: 35Q40

Acknowledgements: The second author is supported by the National Science Foundation under grant DMS-9123208.

*Department of Mathematics, Northwestern University, Evanston, Il 60208 and Department of Mathematics, Stanford University, Stanford, CA 94305

${ }^{\dagger}$ Department of Mathematics, Northwestern University, Evanston, Il 60208 


\section{Introduction}

The quantum hydrodynamic (QHD) model is a moment model, derived from the Wigner equation. It may be viewed as a quantum corrected version of the classical hydrodynamic equations, with the stress tensor and the energy density corrected by $O\left(\hbar^{2}\right)$ perturbations. Some applications, such as the resonant tunnel diode, also involve quantum well potentials. Ancona, Iafrate, and Tiersten ([2], [1]) derived the expression for the stress tensor, and Grubin and Kreskovsky formulated a one dimensional version of the model [11]. A derivation of the general model is carried out by Gardner in [8]. It is of considerable physical and practical importance. An example of this is furnished by the regions of negative differential resistance detected in the voltage current curve associated with the model; closely tied to this is an hysteresis effect, which has been confirmed by Chen, Cockburn, Gardner, and Jerome in [3]. Earlier, Kluksdahl, Kriman, Ferry, and Ringhofer [13] had discovered this effect in the Wigner equation. The QHD model is computationally more tractable than the Wigner formulation, however.

No complete existence theory is available for the classical or quantum hydrodynamic model for semiconductors, either in steady-state or in long time evolution. The model must be self-consistent in the sense that the electric field, which forms a forcing term in the momentum equation, is determined 
by the coupled Poisson equation. The classical isentropic model (or, better, the relaxation perturbation of this model) has been studied self-consistently by Gamba [6] in steady-state and by Zhang [15] in evolution. Their methods are completely different. Gamba employs artificial viscosity, while Zhang proves the convergence of Godunov's method. Although these reduced classical models are somewhat idealized in that heat conduction is not taken into consideration (see [9] and [5] for the simulation of the full classical model), they are still capable of predicting velocity overshoot. In this paper, we consider the quantum equivalent of such a reduced model, in that we analyze self-consistently the perturbation of the classical isentropic model by the relaxation and quantum stress tensor perturbations in the steady-state case. We do not consider quantum well applications discussed in [3]. The model which we study is of physical importance; in fact, a simplification of our model been characterized as a pure state, single carrier transport model in [11].

Our approach is completely novel to this application area, in that we reduce the system to an integro-differential equation, with a set of boundary conditions, including a nonstandard second order boundary condition, which is equivalent to specifying the quantum potential at the (current) inflow boundary. We are able to obtain 'a priori' estimates and an existence result via the Leray-Schauder fixed point theorem. As noted by Irene Gamba [7], 
the technique we use to obtain our pointwise 'a priori' estimates is analogous to division by the dependent variable, and integration along the streamlines of the Bernoulli function. There is some hope, therefore, of eventually extending these results to two spatial dimensions.

The quantum perturbation introduces a third order density perturbation in the momentum equation. Our estimates clearly show that an $\hbar \rightarrow 0$ strong limit is not possible. This is reminiscent, for systems, of the oscillatory dispersion phenomenon noticed by Lax and Levermore in [14]. Their fundamental model was the evolution equation, for which a weak limit was demonstrated and studied. It is not clear whether a weak limit can be demonstrated for this steady-state model; as noted earlier, the limiting equations do have a weak solution [6].

\section{Formulation and Summary of Result}

We shall present the equations for the simplified QHD model as developed in [11] and [8]:

$$
\begin{aligned}
& \rho_{t}+(\rho v)_{x}=0 \\
& (m \rho v)_{t}+\left(m \rho v^{2}+p(\rho)+Q(\rho)\right)_{x}=-\rho \phi_{x}-\frac{m \rho v}{\tau}, \\
& \phi_{x x}=e\left(N_{D}-N_{A}-\rho\right)
\end{aligned}
$$


where $\rho$ is the electron density, $v$ the velocity, and $\phi$ the electrostatic potential. The pressure function, $p=p(\rho)$, has the property that $\rho^{2} p^{\prime}(\rho)$ is strictly monotonically increasing from $[0, \infty)$ onto $[0, \infty)$. A commonly-used hypothesis is [4]:

$$
p(\rho)=k \rho^{\gamma}, \quad \gamma>1, \quad k>0 .
$$

Quantum mechanics is represented by the quantum potential [1]:

$$
Q(\rho)=-\frac{\hbar^{2}}{12 m} \rho(\log (\rho))_{x x},
$$

where $\hbar$ is the normalized (by $2 \pi$ ) Planck's constant and $m$ the effective electron mass. The classical collision term is modeled by the momentum relaxation time approximation, $\tau=\tau(\rho, \rho v)$ in (2). Assume that $\tau$ satisfies

$$
\tau(\rho, \rho v) \geq \tau_{0}>0 .
$$

The device domain is the $x$-interval, $I \equiv(0,1)$. The given functions $N_{D}$ and $N_{A}$ are called the density of donors and the density of acceptors, respectively. They satisfy

$$
N_{D}, N_{A} \in L^{\infty}(I), \quad N_{D}-N_{A} \not \equiv 0 .
$$

The constant $e>0$ in (3) is the electronic charge unit.

In this paper we investigate the steady-state case $\rho_{t}=(\rho v)_{t}=0$. Then, after the introduction of the current density $j=\rho v$, the system (1)-(3) 
reduces to

$$
\begin{aligned}
& j(x)=\text { const. } \\
& \left(\frac{m j^{2}}{\rho}+p(\rho)+Q(\rho)\right)_{x}=-\rho \phi_{x}-\frac{m j}{\tau}, \\
& \phi_{x x}=e\left(N_{D}-N_{A}-\rho\right) .
\end{aligned}
$$

Assume that $j$ is a specified positive constant. Since (9) is a third order ordinary differential equation, and (10) is Poisson's equation, three boundary conditions for (9) and two boundary conditions for (10) are prescribed as follows:

$$
\begin{aligned}
& \rho(0)=\rho_{0}, \quad \rho(1)=\rho_{1}, \quad \rho_{0} \rho_{x x}(0)-\frac{1}{2} \rho_{x}^{2}(0)=\rho_{2}, \\
& \phi(0)=\phi_{0}, \quad \phi(1)=\phi_{1},
\end{aligned}
$$

where $\rho_{0}, \rho_{1}$, and $\rho_{2}$ are positive constants; $\phi_{0}$ and $\phi_{1}$ are the applied bias potentials.

The main result of this paper is as follows.

\section{Theorem 1 Assume that}

$\phi_{1} \geq \phi_{0}+\frac{e}{2}\left\|N_{D}-N_{A}\right\|_{L^{\infty}}, \quad \rho_{2} \geq \frac{12 m}{\hbar^{2}}\left(\frac{m j^{2}}{2}+\frac{k \gamma}{\gamma-1} \rho_{0}^{\gamma+1}\right), \quad\left\|\frac{\partial \tau}{\partial \rho}\right\|_{L^{\infty}} \leq M$

Then, for each $j>0$, there exists a classical solution $(\rho, \phi)$ of (9)-(12), satifying the properties that $\rho \in C_{B}^{3}(I)$ and $\frac{1}{N} \leq \sqrt{\rho} \leq N$ for a positive constant $N$. 
In order to prove the above theorem, we first use a Green's function to solve Poisson's equation (10), and then we reduce the system (9)-(10) to an integro-differential equation. We shall see that the existence of a smooth solution of the original system is equivalent to that of a smooth solution of the integro-differential equation.

The solution of (10) with boundary data (12) is given uniquely by

$$
\phi=e \int_{0}^{1} G(x, \xi)\left(N_{D}-N_{A}-\rho\right) d \xi+x\left(\phi_{1}-\phi_{0}\right)+\phi_{0},
$$

where $G(x, \xi)$ is the Green's function for this problem, and is defined by

$$
G(x, \xi)= \begin{cases}x(\xi-1), & x<\xi \\ \xi(x-1), & x>\xi\end{cases}
$$

We now transform the equation (9) to a second order ordinary differential equation by integration. Dividing (9) by $\rho$, we have

$$
-\frac{1}{\rho}(Q(\rho))_{x}=\left(\frac{m j^{2}}{2 \rho^{2}}+\frac{k \gamma}{\gamma-1} \rho^{\gamma-1}+\phi\right)_{x}+\frac{m j}{\rho \tau} .
$$

Since

$$
\begin{aligned}
-\frac{1}{\rho}(Q(\rho))_{x} & =\frac{\hbar^{2}}{12 m}\left((\log (\rho))_{x}(\log (\rho))_{x x}+(\log (\rho))_{x x x}\right) \\
& =\frac{\hbar^{2}}{12 m}\left(\frac{1}{2}(\log (\rho))_{x}^{2}+(\log (\rho))_{x x}\right)_{x} \\
& =\frac{\hbar^{2}}{12 m}\left(\frac{1}{\sqrt{\rho}}\left(\frac{\rho_{x x}}{\sqrt{\rho}}-\frac{\rho_{x}^{2}}{2 \sqrt{\rho^{3}}}\right)\right)_{x} \\
& =\frac{\hbar^{2}}{6 m}\left(\frac{1}{\sqrt{\rho}}(\sqrt{\rho})_{x x}\right)_{x} .
\end{aligned}
$$


Then, (15) becomes

$$
\frac{\hbar^{2}}{6 m}\left(\frac{1}{\sqrt{\rho}}(\sqrt{\rho})_{x x}\right)_{x}=\left(\frac{m j^{2}}{2 \rho^{2}}+\frac{k \gamma}{\gamma-1} \rho^{\gamma-1}+\phi\right)_{x}+\frac{m j}{\rho \tau} .
$$

Integrating (16) from 0 to $x$ and using the boundary data (11), we have

$$
\begin{aligned}
\frac{\hbar^{2}}{6 m}\left(\frac{1}{\sqrt{\rho}}(\sqrt{\rho})_{x x}\right)= & \frac{m j^{2}}{2 \rho^{2}}+\frac{k \gamma}{\gamma-1} \rho^{\gamma-1}+m j \int_{0}^{x} \frac{d y}{\rho \tau}+\phi \\
& +\frac{\hbar^{2}}{12 m} \frac{\rho_{2}}{\rho_{0}^{2}}-\frac{m j^{2}}{2 \rho_{0}^{2}}-\frac{k \gamma}{\gamma-1} \rho_{0}^{\gamma-1}-\phi_{0}
\end{aligned}
$$

Let $w=\sqrt{\rho}$. By substituting (13) for $\phi$ in (17), the system of equations (8)-(10) reduces to an integro-differential equation with Dirichlet conditions:

$$
\begin{aligned}
& \frac{\hbar^{2}}{6 m} w_{x x}=\frac{m j^{2}}{2 w^{3}}+\frac{k \gamma}{\gamma-1} w^{2 \gamma-1}+m j w \int_{0}^{x} \frac{d y}{w^{2} \tau} \\
& \quad+w\left(e \int_{0}^{1} G(x, \xi)\left(N_{D}-N_{A}-w^{2}\right) d \xi+x\left(\phi_{1}-\phi_{0}\right)+b\right), \\
& w(0)=w_{0}, \quad w(1)=w_{1},
\end{aligned}
$$

where

$$
b=\frac{\hbar^{2}}{12 m} \frac{\rho_{2}}{\rho_{0}^{2}}-\frac{m j^{2}}{2 \rho_{0}^{2}}-\frac{k \gamma}{\gamma-1} \rho_{0}^{\gamma-1}, w_{0}=\sqrt{\rho_{0}}, w_{1}=\sqrt{\rho_{1}} .
$$

If $w$ is a smooth solution of (18)-(19), then

$$
2 w_{0}^{3} w_{x x}(0)=\rho_{2}
$$

That is, the third boundary condition of (11) holds for $w$. Hence, the existence of a smooth solution of (8)-(12) is equivalent to that of a smooth solution of (18)-(19), provided $\rho$ does not vanish on $I$. 


\section{A Priori Estimates and Proof of Theorem}

In this section we study the problem (18)-(19), since it is equivalent to (8)(12), for the existence of smooth solutions. First, we show, in the following lemma, that any solution $w$ of (18)-(19) is nonnegative.

Lemma 1 Assume that the boundary data and given function satisfy the following:

$$
\phi_{1} \geq \phi_{0}+\frac{e}{2}\left\|N_{D}-N_{A}\right\|_{L^{\infty}}, \quad \rho_{2} \geq \frac{12 m}{\hbar^{2}}\left(\frac{m j^{2}}{2}+\frac{k \gamma}{\gamma-1} \rho_{0}^{\gamma+1}\right) .
$$

Then, any solution $w$ of (18)-(19) is nonnegative.

\section{$\underline{\text { Proof Let }}$}

$$
\eta(x)=\max \{-w(x), 0\}
$$

Then, $\eta \in H_{0}^{1}(I)$. Also, $\eta_{x}=-w_{x}$ on the set $A(0)=\{x \in I:-w(x) \geq 0\}$, whereas $\eta_{x}=0$ elsewhere.

Multiplying (18) by $\eta(x)$ and integrating over $(0,1)$, we have

$$
\begin{aligned}
& \frac{\hbar^{2}}{6 m} \int_{0}^{1} \eta_{x}^{2} d x=\int_{0}^{1} w \eta\left(e \int_{0}^{1} G(x, \xi)\left(N_{D}-N_{A}\right) d \xi+x\left(\phi_{1}-\phi_{0}\right)+b\right) d x \\
& \quad+\int_{0}^{1} w \eta\left(\frac{m j^{2}}{2 w^{4}}+\frac{k \gamma w^{2(\gamma-1)}}{\gamma-1}+\int_{0}^{x} \frac{m j}{w^{2} \tau} d y\right) d x \\
& \quad-e \int_{0}^{1} w \eta\left(\int_{0}^{1} G w^{2} d \xi\right) d x
\end{aligned}
$$


From the first condition in (20), we have

$$
\begin{aligned}
& e \int_{0}^{1} G\left(N_{D}-N_{A}\right) d \xi+x\left(\phi_{1}-\phi_{0}\right) \\
& \geq-e\left\|N_{D}-N_{A}\right\|_{L^{\infty}} \int_{0}^{1}|G(x, \xi)| d \xi+x\left(\phi_{1}-\phi_{0}\right) \\
& =x\left(\phi_{1}-\phi_{0}-\frac{e(1-x)}{2}\left\|N_{D}-N_{A}\right\|_{L^{\infty}}\right) \\
& \geq x\left(\phi_{1}-\phi_{0}-\frac{e}{2}\left\|N_{D}-N_{A}\right\|_{L^{\infty}}\right) \\
& \geq 0 .
\end{aligned}
$$

Note that

$$
-G(x, \xi)>0, \quad(x, \xi) \in I \times I .
$$

Thus,

$$
\int_{0}^{1}-G(x, \xi) w^{2} d \xi \geq 0 .
$$

Moreover, by the second condition in (20), $b \geq 0$. Therefore, (21) implies that $\eta=0$; i.e., $w \geq 0$. Q.E.D.

Lemma 2 Let $f(z), \ell_{0} \leq z<\infty$, be nonnegative and nonincreasing such that

$$
f\left(\ell_{2}\right) \leq \frac{C}{\left(\ell_{2}-\ell_{1}\right)^{\alpha}}\left[f\left(\ell_{1}\right)\right]^{\beta}, \quad \ell_{0} \leq \ell_{1}<\ell_{2},
$$

where $C, \alpha$, and $\beta$ are positive constants with $\beta>1$. Then

$$
f\left(\ell_{0}+d\right)=0,
$$

where

$$
d^{\alpha}=C 2^{\frac{\alpha \beta}{\beta-1}}\left[f\left(\ell_{0}\right)\right]^{\beta-1} .
$$


For the proof, see [12, Lemma B.1, p. 63]. An 'a priori' upper bound is obtained in the following lemma.

Lemma 3 Assume that (6), (7), and (20) hold. Then, there exists a positive constant $D$, depending only on the boundary data, such that

$$
w(x) \leq D \text { a.e. in }[0,1] .
$$

Proof For $\ell \geq \ell_{0}=\max \left\{w_{0}, w_{1}\right\}$, define

$$
\eta(x)=\max \{w(x)-\ell, 0\} .
$$

Then, $\eta(x) \geq 0$ and $\eta \in H_{0}^{1}(I)$. Note that $\eta_{x}=w_{x}$ on the set $A(\ell)=\{x \in$ $I ; w(x) \geq \ell\}$, whereas $\eta_{x}=0$ elsewhere.

Multiplying both sides of (18) by $\eta(x)$ and integrating over $(0,1)$, we have

$$
\begin{aligned}
& \frac{\hbar^{2}}{6 m} \int_{0}^{1} \eta_{x}^{2} d x+\frac{k \gamma}{\gamma-1} \int_{0}^{1} w^{2 \gamma-1} \eta d x \\
& +\int_{0}^{1}\left(\frac{m j^{2}}{2 w^{3}}+w \int_{0}^{x} \frac{m j}{w^{2} \tau} d y-e w \int_{0}^{1} G(x, \xi) w^{2} d \xi\right) \eta d x \\
& \leq \int_{0}^{1}\left(e \int_{0}^{1}\left|G\left(N_{D}-N_{A}\right)\right| d \xi+x\left(\phi_{1}-\phi_{0}\right)+b\right) w \eta d x \\
& \leq C_{1} \int_{0}^{1} w \eta d x \\
& \leq C_{1}\left(\int_{0}^{1} w^{2 \gamma-1} \eta d x\right)^{\frac{1}{2 \gamma-1}}\left(\int_{0}^{1} \eta d x\right)^{\frac{2 \gamma-2}{2 \gamma-1}} \\
& \leq \frac{k \gamma}{2(\gamma-1)} \int_{0}^{1} w^{2 \gamma-1} \eta d x+C_{2} \int_{0}^{1} \eta d x,
\end{aligned}
$$


where

$$
\begin{aligned}
C_{1} & =\frac{e}{8}\left\|N_{D}-N_{A}\right\|_{L^{\infty}}+\phi_{1}-\phi_{0}+b, \\
C_{2} & =\frac{2(\gamma-1)}{2 \gamma-1}\left(\frac{2(\gamma-1)}{k \gamma(2 \gamma-1)}\right)^{\frac{1}{2 \gamma-1}} C_{1}^{\frac{2 \gamma-1}{2(\gamma-1)}} .
\end{aligned}
$$

Then, by the Hölder and Poincaré inequalities,

$$
\begin{aligned}
\int_{0}^{1} \eta_{x}^{2} d x & \leq \frac{6 m C_{2}}{\hbar^{2}} \int_{0}^{1} \eta d x \\
& =\frac{6 m C_{2}}{\hbar^{2}} \int_{A(\ell)} \eta d x \\
& \leq \frac{6 m C_{2}}{\hbar^{2}}[\text { meas } A(\ell)]^{\frac{1}{2}}\left(\int_{0}^{1} \eta^{2} d x\right)^{\frac{1}{2}} \\
& \leq \frac{1}{2} \int_{0}^{1} \eta_{x}^{2} d x+\frac{18 m^{2} C_{2}^{2}}{\hbar^{4}}[\text { meas } A(\ell)] .
\end{aligned}
$$

Thus, for $C_{3}=36 \mathrm{~m}^{2} C_{2}^{2}$,

$$
\|\eta\|_{H_{0}^{1}(I)}^{2} \leq \frac{C_{3}}{\hbar^{4}}[\text { meas } A(\ell)] .
$$

From this inequality and Sobolev's inequality, we have, for a fixed constant $\overline{C_{n}}$ and $C_{4}=\overline{C_{n}} C_{3}$,

$$
\begin{aligned}
& \left(\int_{A(\ell)}(w-\ell)^{n} d x\right)^{\frac{2}{n}}=\left(\int_{0}^{1} \eta^{n} d x\right)^{\frac{2}{n}} \\
& \leq \overline{C_{n}}\|\eta\|_{H_{0}^{1}(I)}^{2} \leq \frac{C_{4}}{\hbar^{4}}[\text { meas } A(\ell)]
\end{aligned}
$$

for every positive integer $n \geq 1$.

$$
\begin{aligned}
& \text { If } \ell_{0} \leq \ell_{1}<\ell_{2} \text {, then } A\left(\ell_{2}\right) \subset A\left(\ell_{1}\right) \text { so that } \\
& \qquad\left(\ell_{2}-\ell_{1}\right)^{2}\left[\text { meas } A\left(\ell_{2}\right)\right]^{\frac{2}{n}} \leq\left(\int_{A\left(\ell_{2}\right)}\left(w-\ell_{1}\right)^{n} d x\right)^{\frac{2}{n}}
\end{aligned}
$$




$$
\leq\left(\int_{A\left(\ell_{1}\right)}\left(w-\ell_{1}\right)^{n} d x\right)^{\frac{2}{n}}
$$

Consequently,

$$
\left(\ell_{2}-\ell_{1}\right)^{2}\left[\text { meas } A\left(\ell_{2}\right)\right]^{\frac{2}{n}} \leq \frac{C_{4}}{\hbar^{4}}\left[\text { meas } A\left(\ell_{1}\right)\right]
$$

That is,

$$
\text { meas } A\left(\ell_{2}\right) \leq\left(\frac{\sqrt{C}_{4}}{\left(\ell_{2}-\ell_{1}\right) \hbar^{2}}\right)^{n}\left[\text { meas } A\left(\ell_{1}\right)\right]^{\frac{n}{2}} \text {. }
$$

We take $n>2$ and apply Lemma 2 to derive that

$$
\text { meas } A(D)=0 \text {, }
$$

where

$$
D=\frac{\sqrt{C_{4}} 2^{\frac{n}{n-2}}}{\hbar^{2}}+\ell_{0}
$$

This implies that $w \leq D$ a.e. in $[0,1]$. Q.E.D.

We have already demonstrated that solutions $w$ are necessarily nonnegative. A positive 'a priori' lower bound is proven next.

Lemma 4 Assume that the conditions of Lemma 3 hold. Then, there exists a constant $\delta>0$ such that

$$
w(x) \geq \delta>0, \quad x \in[0,1]
$$

where $\delta$ is independent of $w$. 
Proof Assume that there were not a positive constant $\delta$ such that $w(x) \geq$ $\delta>0$ for $x \in[0,1]$. Then, there exist a sequence of solutions $\left\{w_{n}\right\}$ of (18)-(19) and a sequence $\left\{x_{n}\right\} \subset(0,1)$ such that $w_{n}\left(x_{n}\right) \rightarrow 0$ as $n \rightarrow \infty$.

We claim that the sequence $\left\{w_{n}\left(x_{n}\right)\right\}$ goes to zero exponentially. By the condition (20) in Lemma 1, solutions $w_{n}$ of (18) satisfy the following inequality

$$
\lambda_{1}\left(w_{n}\right) w_{n} \leq\left(w_{n}\right)_{x x},
$$

where

$$
\lambda_{1}\left(w_{n}\right)=\frac{3 m^{2} j^{2}}{\hbar^{2}\left(w_{n}\right)^{4}} .
$$

Consider the following linear equation:

$$
\left\{\begin{array}{l}
w_{x x}=\lambda_{1}(\bar{w}) w \\
w(0)=w_{0}, \quad w(1)=w_{1},
\end{array}\right.
$$

where $\bar{w}$ is a constant satisfying $0<\bar{w} \leq D$. The solution of the above equation is

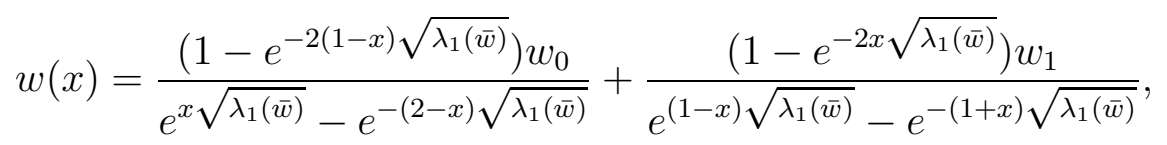

which satisfies the inequality,

$$
w(x) \leq \frac{w_{0}}{e^{x \sqrt{\lambda_{1}(\bar{w})}}-e^{-(2-x) \sqrt{\lambda_{1}(\bar{w})}}}+\frac{w_{1}}{e^{(1-x) \sqrt{\lambda_{1}(\bar{w})}}-e^{-(1+x) \sqrt{\lambda_{1}(\bar{w})}}} .
$$

Let $\bar{w}=w_{n}\left(x_{n}\right)$ in (25). Then the solutions represented by (25) decay to zero exponentially as $n \rightarrow \infty$. By (24) and the maximum principle, $\left\{w_{n}\left(x_{n}\right)\right\}$ decays also to zero exponentially. 
Using the Green's function defined by (14), we have an implicit expression for the solution $w_{n}$ of (18)-(19):

$$
\begin{aligned}
& \frac{\hbar^{2}}{6 m} w_{n}(x)=\frac{m j^{2}}{2} \int_{0}^{1} \frac{G(x, \xi)}{\left(w_{n}(\xi)\right)^{3}} d \xi+\int_{0}^{1} G w_{n}(\xi)\left(\int_{0}^{\xi} \frac{m j}{w_{n}^{2} \tau} d y\right) d \xi \\
& \quad+e \int_{0}^{1} G w_{n}(\xi)\left(\int_{0}^{1} G\left(\xi, \xi_{1}\right)\left(N_{D}-N_{A}-w_{n}^{2}\right) d \xi_{1}+\xi\left(\phi_{1}-\phi_{0}\right)+b\right) d \xi \\
& \quad+\int_{0}^{1} G \frac{k \gamma w_{n}^{2 \gamma-1}}{\gamma-1} d \xi+x\left(w_{1}-w_{0}\right)+w_{0} .
\end{aligned}
$$

By Lemma 3 and the fact that $G(x, \xi)<0$ for $(x, \xi) \in I \times I$, we have

$$
w_{n}\left(x_{n}\right) \leq \frac{3 m^{2} j^{2}}{\hbar^{2}} \int_{0}^{1} \frac{G\left(x_{n}, \xi\right)}{\left(w_{n}(\xi)\right)^{3}} d \xi+C(D)
$$

Since $G\left(x_{n}, \xi\right)$ is a piecewise linear function defined by (14), and $\left\{\frac{1}{w_{n}\left(x_{n}\right)}\right\}$ goes to $\infty$ exponentially, the improper integral of (26) diverges to $-\infty$. Thus, by (26), there exists a positive integer $N$ such that $w_{n}\left(x_{n}\right)<0$ when $n \geq N$. This is impossible because of Lemma 1.

Q.E.D.

The final estimate is an $L_{2}$ bound for the fourth derivative of $w$.

Lemma 5 Assume that the conditions of Lemma 3 hold and the relaxation time $\tau$ satisfies

$$
\left\|\frac{\partial \tau}{\partial w}\right\|_{L^{\infty}} \leq M
$$

Then, there exists a constant $C=C(\delta, D)$ such that

$$
\int_{0}^{1} w_{x x x x}^{2} d x \leq \frac{C}{\hbar^{4}},
$$


where $C$ is independent of $w$.

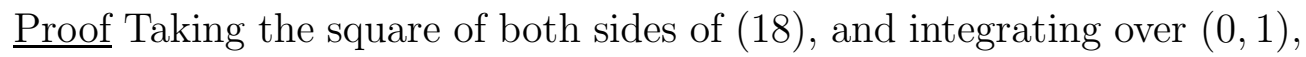
we have

$$
\frac{\hbar^{4}}{36 m^{2}} \int_{0}^{1} w_{x x}^{2} d x=\int_{0}^{1} g^{2}(w) d x
$$

where $g(w)$ is the right hand side of (18); i.e.,

$$
\begin{aligned}
g(w)= & \frac{m j^{2}}{2 w^{3}}+\frac{k \gamma}{\gamma-1} w^{2 \gamma-1}+w \int_{0}^{x} \frac{m j}{w^{2} \tau} d y \\
& +w\left(e \int_{0}^{1} G(x, \xi)\left(N_{D}-N_{A}-w^{2}\right) d \xi+x\left(\phi_{1}-\phi_{0}\right)+b\right) .
\end{aligned}
$$

It follows from Lemma 3 and Lemma 4 that

$$
\int_{0}^{1} w_{x x}^{2} d x \leq \frac{C_{1}}{\hbar^{4}}
$$

where $C_{1}=C_{1}(\delta, D)$ is independent of $w$.

Taking the second derivative of both sides of (18) and multilying by $w_{x x x x}$, we have

$$
\frac{\hbar^{2}}{6 m} \int_{0}^{1} w_{x x x x}^{2} d x=I_{1}+I_{2}+I_{3}+I_{4}
$$

where

$$
\begin{aligned}
I_{1}=\int_{0}^{1} & \left(-\frac{3 m j}{2 w^{4}}+\frac{k \gamma(2 \gamma-1)}{\gamma-1} w^{2(\gamma-1)}+x\left(\phi_{1}-\phi_{0}\right)+b\right) w_{x x} w_{x x x x} d x \\
& +\int_{0}^{1}\left(\int_{0}^{x} \frac{m j}{w^{2} \tau} d y+e \int_{0}^{1} G\left(N_{D}-N_{A}-w^{2}\right) d \xi\right) w_{x x} w_{x x x x} d x \\
I_{2}= & \int_{0}^{1}\left(\frac{6 m j^{2}}{w^{5}}+2 k \gamma(2 \gamma-1) w^{2 \gamma-3}\right) w_{x}^{2} w_{x x x x} d x
\end{aligned}
$$




$$
\begin{aligned}
& I_{3}=2 \int_{0}^{1}\left(\phi_{1}-\phi_{0}-\frac{m j \tau^{\prime}}{\tau^{2}}+e \int_{0}^{1} G_{x}\left(N_{D}-N_{A}-w^{2}\right) d \xi\right) w_{x} w_{x x x x} d x \\
& I_{4}=e \int_{0}^{1}\left(N_{D}-N_{A}-w^{2}\right) w w_{x x x x} d x
\end{aligned}
$$

It follows from $(22),(23),(6)$, and (28) that

$$
\begin{aligned}
& \left|I_{1}\right|+\left|I_{3}\right|+\left|I_{4}\right| \leq \frac{\hbar^{2}}{24 m} \int_{0}^{1} w_{x x x x}^{2} d x+C_{2}(\delta, D) \int_{0}^{1}\left(w_{x x}^{2}+w_{x}^{2}+w^{2}\right) d x \\
& \leq \frac{\hbar^{2}}{24 m} \int_{0}^{1} w_{x x x x}^{2} d x+\frac{C_{3}(\delta, D)}{\hbar^{4}}
\end{aligned}
$$

Recall the interpolation inequality,

$$
w_{x}^{2} \leq \nu \int_{0}^{1} w_{x x}^{2} d x+C_{\nu} \int_{0}^{1} w^{2} d x
$$

As a consequence of this inequality and (28), we have

$$
\left|I_{2}\right| \leq \frac{\hbar^{2}}{24 m} \int_{0}^{1} w_{x x x x}^{2} d x+\frac{C_{4}(\delta, D)}{\hbar^{2}} .
$$

Thus,

$$
\int_{0}^{1} w_{x x x x}^{2} d x \leq \frac{C(\delta, D)}{\hbar^{4}}
$$

where $C$ is independent of $w$.

Q.E.D.

Some comments are now in order. From Lemma 5, we conclude $w \in H^{4}(I)$ lies in a set with 'a priori' bound. Thus, by the Sobolev embedding theorem, $w \in C_{B}^{3}(I)$ also lies in a set with 'a priori' bound. Moreover, by Lemma 3 and Lemma 4 , there exists a constant $N$, depending only on the boundary data, such that

$$
\frac{1}{N} \leq w(x) \leq N, \quad x \in[0,1]
$$


The following Lemma is a special case of the Leray-Schauder fixed point theorem. For the proof, see [10, Theorem 10.3, p. 222].

Lemma 6 Let $T$ be a compact mapping of a Banach space $B$ into itself. Suppose there exists a constant $M$ such that

$$
\|u\|_{B} \leq M
$$

for all $(u, \sigma) \in B \times[0,1]$ satisfying $u=T(u, \sigma)$. Then $T(u, 1)$ has a fixed point.

We are now prepared to give the final arguments.

Lemma 7 Assume that the conditions of Theorem 1 hold. Then, there exists a solution of (18)-(19).

$\underline{\text { Proof }}$ To apply Lemma 6 , we construct an operator $T_{\alpha}: C^{0,1}[0,1] \rightarrow$ $C^{0,1}[0,1]$ for a fixed constant $\alpha>0$ by solving the linear equation,

$$
\left\{\begin{array}{l}
w_{x x}=\frac{6 m^{2}}{\hbar^{2}} g_{\alpha}(u), \\
w(0)=w_{0}, \quad w(1)=w_{1},
\end{array}\right.
$$

where

$$
g_{\alpha}(u)= \begin{cases}g(u), & u>\alpha, \\ g(\alpha), & u \leq \alpha,\end{cases}
$$

for $u \in C^{0,1}[0,1]$ and $g(u)$ is defined by $(27)$. 
The linear equation (30) is uniformly elliptic. For every function $u \in$ $C^{0,1}[0,1]$, there exists a unique solution $w=T_{\alpha}(u)$ satisfying

$$
\|w\|_{C^{1,1}} \leq M\left(\alpha, N_{1}\right), \quad\|u\|_{C^{0,1}} \leq N_{1} .
$$

That is, $T_{\alpha}$ maps bounded sets of $C^{0,1}[0,1]$ into bounded sets of $C^{1,1}[0,1]$. By the Ascoli-Arzela theorem, $T_{\alpha}$ is compact in $C^{0,1}[0,1]$; continuity is routine.

If $w$ satisfies

$$
\left\{\begin{array}{l}
w_{x x}=\sigma \frac{6 m^{2}}{\hbar^{2}} g_{\alpha}(w) \\
w(0)=w_{0}, \quad w(1)=w_{1},
\end{array}\right.
$$

for $0 \leq \sigma \leq 1$, i.e., $w$ is a solution of $w=\sigma T_{\alpha}(w)$, then it follows from the previous lemmas that $0 \leq w \leq N$ and $\|w\|_{C^{0,1}} \leq N_{1}$. By Lemma 6, we conclude the existence of a fixed point of $T_{\alpha}$; i.e., there exists a $w$ such that

$$
\left\{\begin{array}{l}
w_{x x}=\frac{6 m^{2}}{\hbar^{2}} g_{\alpha}(w) \\
w(0)=w_{0}, \quad w(1)=w_{1} .
\end{array}\right.
$$

By (29), w satisfies

$$
\frac{1}{N} \leq w(x) \leq N, \quad x \in[0,1]
$$

In particular, we take $\alpha<\frac{1}{N}$ in (30) such that $g_{\alpha}(w)=g(w)$ for $\frac{1}{N} \leq w \leq N$. Therefore, such a $w$ solves (18)-(19). $\quad$ Q.E.D.

As a consequence of Lemma 7, Theorem 1 has been proved. 


\section{References}

[1] Ancona M.G. \& Iafrate G.J., Quantum correction to the the equation of state of an electron gas in a semiconductor. Physical Review, B39:9536$9540,1989$.

[2] Ancona M.G. \& Tiersten H.F., Macroscopic physics of the silicon inversion layer. Physical Review, B35:7959-7965, 1987.

[3] Chen Z., Cockburn B., Gardner C.L. \& Jerome J.W., Quantum hydrodynamic simulation of hysteresis in the resonant tunneling diode. IMA Preprint Series \# 1227, Univ. Minnesota, April, 1994, to appear in J. Comp. Phys..

[4] Courant R. \& Friedrichs K.O., Supersonic Flow and Shock-Waves. J. Wiley and Sons, New York, 1967.

[5] Fatemi E., Jerome, J. \& Osher S., Solution of the hydrodynamic device model using high-order nonoscillatory shock capturing algorithms. IEEE Transactions on Computer-Aided Design of Integrated Circuits and Systems, CAD-10:232-244, 1991.

[6] Gamba I.M., Stationary transonic solutions of a one-dimensional hydrodynamic model for semiconductors. Commun. in P.D.E., 17:553-577, (1992). 
[7] Gamba I.M., Personal Communication.

[8] Gardner C.L., The quantum hydrodynamic model for semiconductor devices. SIAM J. Appl. Math., 54:409-427, 1994.

[9] Gardner C.L., Jerome J.W. \& Rose D.J., Numerical methods for the hydrodynamic device model: Subsonic flow. IEEE Transactions on Computer-Aided Design of Integrated Circuits and Systems, CAD-8:501$507,1989$.

[10] Gilbarg D. \& Trudinger N.S., Elliptic Partial Differential Equations of Second Order. Spriger-Verlag, Berlin, 1977.

[11] Grubin H.L. \&Kreskovsky J.P., Quantum moment balance equations and resonant tunnelling structures. Solid-State Electronics, 32:10711075, (1989).

[12] Kinderlehrer D. \& Stampacchia G., An Introduction to Variational Inequalities and Their Applications. Academic Press, New York, 1980.

[13] Kluksdahl N.C., Kriman A.M., Ferry D.K. \& Ringhofer C., Selfconsistent study of the resonant tunneling diode. Physical Review, B39:7720-7735, 1989. 
[14] Lax P.D. \& Levermore C.D., The small dispersion limit for the Korteweg-de Vries equation I. Comm. Pure Appl. Math., 36:253-290, 1983.

[15] Zhang B., Convergence of the Godunov scheme for a simplified onedimensional hydrodynamic model for semiconductor devices. Commun. Math. Phys., 157:1-22, (1993). 